\title{
Aluminum Foil Anodes for Li-ion Rechargeable Batteries: The Role of Li Solubility within $\beta$-LiAl
}

Tianye Zheng a,b, Dominik Kramer ${ }^{\mathrm{c}}$, Reiner Mönig ${ }^{\mathrm{c}}$, Steven T. Boles ${ }^{\mathrm{a}, \mathrm{d}, *}$

${ }^{a}$ Department of Electrical Engineering, The Hong Kong Polytechnic University, Hung Hom, Kowloon, Hong Kong

${ }^{\mathrm{b}}$ Centre for Advances in Reliability and Safety (CAiRS), Hong Kong Science Park, Pak Shek Kok, New Territory, Hong Kong

${ }^{c}$ Institute for Applied Materials, Karlsruhe Institute of Technology, 76344 EggensteinLeopoldshafen, Germany

${ }^{\mathrm{d}}$ Department of Energy and Process Engineering, Norwegian University of Science and Technology, Høgskoleringen 1, 7491 Trondheim, Norway

First author: darren.ty.zheng@connect.polyu.hk

*Corresponding author: steven.t.boles@polyu.edu.hk 


\section{Abstract}

Li-ion battery (LIB) electrodes contain a substantial amount of electrochemically inactive materials, including binder, conductive agent, and current collectors. These extra components significantly dilute the specific capacity of whole electrodes, and thus have led to efforts to utilize foils, e.g., Al, as the sole anode material. Interestingly, the literature has many reports of fast degradation of Al electrodes, where less than a dozen cycles can be achieved. However, in some studies, $\mathrm{Al}$ anodes demonstrate stable cycling life with several hundred cycles. In this work, we present a successful pathway for enabling long-term cycling of simple Al foil anodes: $\beta$-LiAl phase grown from Al foil ( $\alpha$-Al) exhibits a cycling life of 500 cycles with a $\sim 96 \%$ capacity retention when paired with a commercial cathode. The excellent performance stems from strategic utilization of the Li solubility range of $\beta$-LiAl that can be (de-)lithiated without altering its crystal structure. This solubility range at room temperature is determined to be $\sim 6$ at $\%$. Consequently, this design circumvents the critical issues associated with the $\alpha / \beta / \alpha$ phase transformations, such as volume change, mechanical strain, and nanopore formation. Application-wise, the maturity of aluminum industry, combined with excellent sustainability prospects, makes this anode an important option for future devices.

Keywords: lithium-ion battery, solid-state anode, aluminum foil, $\beta$-LiAl, solubility range 


\section{INTRODUCTION}

Aluminum has been explored as a candidate for the negative electrode in lithium-based rechargeable batteries since the 1970s. ${ }^{1}$ Generally, investigations of this system center around the phase transformations between the $\alpha$ phase (fcc, Al) and the $\beta$ phase (cubic, LiAl), which correspond to a high theoretical capacity of $\sim 993 \mathrm{mAh} \mathrm{g}^{-1}$ at room temperature. Efforts were made to utilize and to understand this Li-Al electrode until Sony introduced the Li-ion battery (LIB) with graphite as the negative electrode in the early 1990s. ${ }^{2-3}$ During the past decade, there has been a strong shift of focus onto alloy anode candidates which achieve some of the highest absolute specific capacity figures, such as silicon $(\mathrm{Si})$, tin $(\mathrm{Sn})$, and germanium $(\mathrm{Ge}) .{ }^{4-5}$ To date, only limited success has been attained for Al-based anodes due to the issues that are not yet resolved, including significant mechanical strain, ${ }^{6}$ brittleness of the $\beta$-LiAl,${ }^{7-8}$ and electrode pulverization during delithiation. ${ }^{9-10}$ Our previous study suggests that the $\mathrm{Al} / \mathrm{LiAl} / \mathrm{Al}(\alpha / \beta / \alpha)$ phase transformations might be intrinsically challenging to utilize due to the formation of nanopores, which cause a loss of electrolyte due to secondary SEI formation on the large surface area. Therefore, a strategic pathway is required to mitigate degradation. ${ }^{9}$ From a more holistic perspective, conventional composite anodes may be ill-suited to wide-spread energy storage efforts if cradle-to-grave costs and sustainability are considered. In today's state-of-the-art devices, both the cathode and the anode consist of composites of active materials, polymer binders and conductive additives. These components are dispersed into a solvent to form a "slurry", which is then pasted onto the current collectors (e.g., copper foil for the anodes) for drying. Not only do the 
toxicity of some chemicals present risks for occupational safety and for the environment, but also the multi-step nature of the electrode manufacturing requires significant labor and capital at scale. Similarly, the complexity of recycling and disposal increases as the mixing of materials in cells becomes increasingly significant. This electrode architecture also reduces the specific capacity, particularly because the copper current collector is electrochemically inactive, yet has a relatively high density as a material. ${ }^{11}$ This mass of the copper current collector is often neglected in academic discussions and numbers reported for 'high-performance' anode materials are usually normalized to the active material only. ${ }^{12}$

In this study, a novel anode structure has been developed by partly lithiating a metallic Al foil to form a monolithic electrode. Although this prelithiation step is done electrochemically here, other methods like a simple mechanical rolling, will also be sufficient to fabricate such an electrode. ${ }^{13}$ The $\beta$-LiAl and the $\alpha$-Al layers function as the active material and the current collector, respectively. This design significantly simplifies the electrode manufacturing and reduces fabrication costs by omitting the usage of copper foils and binders. Replacing the graphite anode with such an Al-based electrode, may result in a considerable reduction in material costs for LIB since the costs of conventional copper and carbon-based anodes are roughly one fifth the cell costs. ${ }^{14}$ Consequently, the baking and calendering processes needed for conventional composite electyrode coatings, which can be quite resource-consuming (e.g. energy/yield), are also omitted. Lastly, the metallurgical nature of this electrode design may potentially have a 
positive impact on the sustainability prospects of such batteries, as the composite nature of waste cells is reduced or eliminated.

To enable the $\beta$ - $\mathrm{LiAl}$ on $\mathrm{Al}$ layered structure as a stable anode, a new concept for cycling the $\beta$-LiAl solely within its Li solubility range and without a phase transition (i.e., maintaining the $\beta$ phase crystal structure) is further proposed. Although a Li solubility range within the $\beta$ phase regime is included in the $\mathrm{Li}-\mathrm{Al}$ phase diagrams, the solubility range accessible at low temperatures has never been specified due to the experimental conditions of previous phase diagram studies made above $400{ }^{\circ} \mathrm{C}$. Different extrapolations to room temperature have been suggested, they range from $9.2 \%$ at $423^{\circ} \mathrm{C}$ which might be maintained at room temperature ${ }^{15}$ to a solubility range decreasing with temperature, being less than one percent (ca. $49.2 \%$ to 50.1 at $\% \mathrm{Li}$ ) below $100^{\circ} \mathrm{C} .{ }^{16}$ To the best of our knowledge, no study has determined the solubility range at room temperature and the related capacity. For electrochemical lithiation of Al, the vast majority of studies focus on the $\beta$-LiAl and neglect the phases with higher Li content, because these phases necessitate very low potentials and elevated temperatures. ${ }^{17}$ Therefore, it is believed that $\beta$-LiAl is the only relevant phase based on the first cycle capacity and x-ray data, even though trace amounts of Li-rich phases may exist, e.g., on the electrode surface. ${ }^{18}$

Only a few studies have demonstrated reasonable cycling performance for Al-based anodes, e.g., a demonstration of 250 cycles with $>80 \%$ capacity retention. ${ }^{19}$ Others have found that mechanical strain caused by (de-)alloying with Li is problematic for $\mathrm{Al}$ anodes, and the capacity loss can be limited to less than $12 \%$ after 200 cycles for a full 
cell paired with a $\mathrm{LiFePO}_{4}$ (LFP) cathode, by uniformly distributing the mechanical stress generated during (de-)lithiation. ${ }^{20}$ Similarly, Li et al. claim that a mechanically hard Al foil can minimize the mechanical damage during the phase transition, thus giving 120 stable cycles when paired with a $\mathrm{LiCoO}_{2}$ (LCO) cathode. ${ }^{21}$ Although a mechanically stable structure can indeed contribute to the above reported cycling performances, other beneficial aspects should not be neglected, such as material and interface chemistries. At end of the day, a unified understanding of why Al foil anodes often fail prematurely, but sometimes appear to be adequate is still yet to be achieved. In this work, multiple approaches have been utilized to characterize the solubility range of the $\beta$-LiAl independently, including electrochemical characterizations, ex situ x-ray diffraction, and in situ stress measurements. In addition, scanning electron microscopy (SEM) has been done to observe the morphologies of partly-lithiated Al foils, providing insights into the $\mathrm{Li}-\mathrm{Al}$ system. Based on these observations, it is possible to electrochemically cycle the electrode such that the active layer (i.e., $\beta$-LiAl) of the anode stays within its Li solubility range. Extending the cycled amount of lithium leads to phase boundary motion between alpha and beta and can largely explain the origins of capacity fading in Al-based anodes. Lastly, a full cell is assembled combining $\beta$-LiAl grown on $\mathrm{Al}$ and a commercially available cathode to demonstrate the feasibility of this solid-state anode technology. 


\section{EXPERIMENTAL Methods}

Electrochemical Tests. All electrochemical tests were carried out by a compactstat (Ivium

Technologies, the Netherlands) and a VMP potentiostat (Biologic Technologies, France), including chronoamperometry (CA), galvanostatic charge-discharge (GCD), cyclic voltammetry (CV) and electrochemical impedance spectroscopy (EIS).

Firstly, the charge counting experiment (i.e., CA) was done using $0.25 \mathrm{~mm}$ thick highpurity Al foils (99.9995\%; Alfa Aesar). Swagelok type cells $(\varnothing=11 \mathrm{~mm})$ and were assembled using the $\mathrm{Al}$ foils and the Li foils as the working and the counter electrodes, respectively. A $\mathrm{LiPF}_{6}$ electrolyte (1M; EC:EMC 3:7 vol\%) and a glass fiber separator (Whatman ${ }^{\circledR}$ ) were also utilized to achieve the typical half-cell configuration. Secondly, a typical cyclic voltammogram was obtained using a $20 \mu \mathrm{m} \mathrm{Al} \mathrm{foil} \mathrm{(i.e.,} \mathrm{cathodic} \mathrm{current}$ collector) assembled in a conventional coin cell (half-cell). Thirdly, the CV test series were conducted using $0.1 \mathrm{~mm}$ thick Al foils (99.997\%; Alfa Aesar) under the coin cell architecture. The prelithiation is achieved by firstly polarizing the coin cell at $10 \mathrm{mV}$ vs. $\mathrm{Li} / \mathrm{Li}^{+}$for 15 minutes to achieve a homogenous nuclei distribution among the $\mathrm{Al}$ electrode surface. ${ }^{8}$ The potential was then switched to a moderate level $(150 \mathrm{mV}$ vs. $\left.\mathrm{Li} / \mathrm{Li}^{+}\right)$to facilitate a constant propagation of the phase boundary and to form a homogenous layer of $\beta$-LiAl covering the surface of the Al foil until the desired prelithiation depths are achieved. ${ }^{8}$ For instance, a $20 \mu \mathrm{m}$ prelithiation depth refers to: $0.002 \mathrm{~cm} \times 1 \mathrm{~cm}^{2} \times 2.7 \mathrm{~g} / \mathrm{cm}^{3} \times 993 \mathrm{mAh} / \mathrm{g}=\sim 5.36 \mathrm{mAh} / \mathrm{cm}^{2}$.

Ex Situ X-ray Diffraction (XRD). A deep lithiated $\mathrm{Al}$ electrode (i.e., $5 \mathrm{mV}$ vs. $\mathrm{Li} / \mathrm{Li}^{+}$for 3 
days) underwent an x-ray diffraction using a high-energy Rigaku SmartLab diffractometer equipped with a Mo anode tube. Such a Li-Al electrode was prepared by disassembling a Swagelok cell that underwent a potentiostatic hold at $10 \mathrm{mV}$ vs. $\mathrm{Li} / \mathrm{Li}^{+}$for 3 days and then sealing into Kapton tapes inside an argon-filled glove box.

In Situ Stress Measurement. Cantilevers made of aluminum oxide with the size of $15 \times 5 \times 0.25$ $\mathrm{mm}^{3}$ are double-side polished prior to the similar PVD processes described in a previous study. ${ }^{9}$ The thickness of TiN (current collector) and Al films (electrode material) for stress measurement are characterized to be $\sim 160 \mathrm{~nm}$ and $\sim 420 \mathrm{~nm}$, respectively. The GCD tests were run for the in situ stress cell at a rate and $\mathrm{C} / 10$, determined by the total charge of the Al film. In situ stress measurement was achieved using the method of substrate curvature. In a substrate-based model where a rigid interface exists, the volume expansion caused by Li insertion strains the substrate and results in compressive stresses. The home-built three-electrode cell and two-beam laser setup allow simultaneous measurement of the curvature of the substrate. Once the lithiation starts, the bending of the alumina cantilever can be tracked in situ by recording the distance change between the two laser spots. It should be noted that the stress values are normalized to the initial Al film thickness, referring to the nominal stress, such that the thickness change during (de)lithiation is not taken into consideration. In other words, the mechanical stress reported in this study is a product of stress-thickness over the initial thickness and can be quantified using the Stoney equation (details can be found elsewhere). ${ }^{9}$

Cross-sectional Scanning Electron Microscopy (SEM). The Swagelok cells are also used here. When the Al foil is lithiated/delithiated to the desired state of charge/discharge, the 
Swagelok cell was disassembled in an argon-filled glovebox. The partly lithiated/delithiated Al foils underwent a series of grinding processes using sandpapers from \#1000 to \#5000 to create a flat and smooth cross-section. A thick foil obtained from Alfa Aesar $(99.9995 \% ; 0.25 \mathrm{~mm})$ was used to maximize the cross-sectional area. Electrochemically, the thick foils were lithiated using potentiostatic modes to maintain a constant driving force.

A specifically designed transfer system (Leica VCT100) allows for the immediate sample transfer from the glovebox to the SEM (Zeiss Merlin) without exposure to air. SEM images were acquired under an acceleration voltage of $6 \mathrm{kV}$, using both a SE detector and a BSE detector, such that the three-dimensional (3D) morphology and the $\beta$ phase distribution can be clearly revealed.

Full Cell Performance Assessment. A commercially available LFP cathode was purchased (1 mAh $\mathrm{cm}^{-2}$; CUSTOMCELLS, Germany) and paired with the novel bilayer $\beta$-LiAl anode developed in this study. The cells were examined in a Swagelok type cell $(\varnothing=11 \mathrm{~mm})$, using a porous polymer separator (Celgard, USA) and A LiPF 6 electrolyte (1M, EC:EMC 3:7 vol\%). The current rate equivalent to $\mathrm{C} / 10$ (normalized to the LFP cathode; $0.1 \mathrm{~mA} \mathrm{~cm}^{-2}$ ) is used for cycling performance assessment. 


\section{RESULTS AND DISCUSSION}

During the experimental design, we strategically focus on partial lithiation to circumvent any possible formations of Li-rich phases. While the $\alpha$ phase is present and overall $\alpha / \beta$ equilibrium is maintained, only $\beta$ - $\mathrm{LiAl}$ is formed at relatively high potentials $\left(>0.2 \mathrm{~V}\right.$ vs. $\mathrm{Li} / \mathrm{Li}^{+}$; i.e., lithiation plateau), although an overpotential is required to move the phase front. In this case, the Li solubility within the $\beta$-LiAl can be characterized and discussed in isolation.

\section{Solubility Range Characterized by Electrochemical Techniques}

Figure 1a shows a typical cyclic voltammogram of Al foils against Li metal obtained at a slow scan rate of $0.01 \mathrm{mV} \mathrm{s}^{-1}$, covering a wide potential range from $1.5 \mathrm{~V}$ to $0 \mathrm{~V}$ vs. $\mathrm{Li} / \mathrm{Li}^{+}$. The sharp peaks near $0.2 \mathrm{~V}$ (reduction) and $0.5 \mathrm{~V}$ (oxidation) depict the formation of the $\beta$ - $\mathrm{LiAl}$ and the reformation of the $\alpha$ phase, respectively. Apart from the reactions of alloying and dealloying, there is also a broad but weak peak at $\sim 0.3 \mathrm{~V}$ (blue arrow) that was considered by Hudak et al. as "unexplained". ${ }^{22}$ Furthermore, one can notice that the reduction current is almost zero prior to the lithiation peak while it does not go back to zero after the lithiation peak. Interestingly, the electrical charge integrated from the reduction current after the $\beta$ phase formation peak (i.e., $\sim 0.2 \mathrm{~V}$ to $0 \mathrm{~V} ; 0.826$ $\mathrm{mAh} \mathrm{cm}^{-2}$ ) is the same as the amount integrated from the oxidation current prior to the

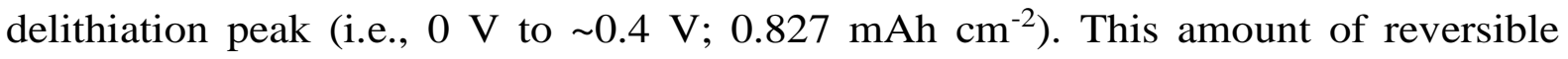
capacity is calcualted to be around $18 \%$ as compared to the one contributed by $\alpha / \beta$ phase transformations (i.e., $\sim 4.7 \mathrm{mAh} \mathrm{cm}^{-2}$ and $\sim 4.5 \mathrm{mAh} \mathrm{cm}^{-2}$ intergated from the lithiation and the delithiation peak, repectively). Such an electrochemical indication suggests that 
some reversible reactions other than $\alpha / \beta$ equilibrium are probably ocurring within these potential ranges, including (de-)lithiation of SEI, formation of Li-rich phases, and (de)saturation of the solubility range of the $\beta$-LiAl.

Electrochemical charge counting was used to examine the composition of lithiated Al $\left(\mathrm{Li}_{\mathrm{x}} \mathrm{Al}\right)$ at room temperature, focusing on the potential range for lithiation (i.e., $\sim 0.3 \mathrm{~V}$ to $0 \mathrm{~V}$ ). The thick Al foils used in this study are well suited for conducting such an experiment because the impact from surface reactions, such as SEI formation and oxide lithiation, are negligible considering the overall thickness $(0.25 \mathrm{~mm}$, any native oxide layer is only present on the surface with a thickness in nm scale). As can be seen from Figure $1 b$, potentiostatic charge counting indicates that the Li content in $\beta$-LiAl varies from 48.9 to 53.7 at. $\%$ after the room temperature lithiation within a potential range between $250 \mathrm{mV}$ and $0 \mathrm{mV}$ vs. $\mathrm{Li} / \mathrm{Li}^{+}$for at least 96 hours $(250 \mathrm{mV}$ for 120 hours). A dashed line displays the Li solubility of the $\beta$ phase and the boundary of a Li-rich $(\gamma)$ phase, previously determined by the coulometric titration method at $415^{\circ} \mathrm{C} .{ }^{23-24} \mathrm{~A}$ sharp decrease of potential was observed by adding a small amount of $\mathrm{Li}$ on the $\mathrm{Li}$-poor side at $415^{\circ} \mathrm{C}$ which corresponds to the large negative slope at low $\mathrm{Li}$ content we obtained at room temperature. On the $\mathrm{Li}$-rich side, the $\beta$ and $\gamma$ phase coexist at $415^{\circ} \mathrm{C}$, exhibiting a potential plateau at $\sim 70 \mathrm{mV}$, for a $\mathrm{Li}$ content beyond $\sim 54$ at. \%. For our charge counting, no indication of the $\gamma$ phase can be seen nor from the phase diagram published in 1982. ${ }^{16}$ Additionally, an XRD test has been done for a deep lithiated Al foil. The obtained diffractogram in Figure S1 only shows the $\beta$-LiAl peaks. Together, crystalline Li-rich phases higher than the $\beta$-LiAl are either absent or minute in the fully lithiated 
foil samples (i.e., amorphous phases can not be excluded). ${ }^{18}$ The present work focuses on cycling within the solubility range. However, the capacity that we determined for the formation of the $\beta$-LiAl phase, $1151.68 \mathrm{mAh} \mathrm{g}^{-1}$, will be relevant for future work aiming at using the full capacity of the $\alpha / \beta$ phase transition: Instead of a theoretical capacity of $993 \mathrm{mAh} \mathrm{g}^{-1}$ calculated for the ideal 50:50 composition $\left(\mathrm{Li}_{1.000} \mathrm{Al}\right)$, the value of 1152 $\mathrm{mAh} \mathrm{g}^{-1}$ should be used as theoretical capacity of the $\beta$ phase at room temperature, to account for the 53.7 at $\%$ of $\mathrm{Li}\left(\mathrm{Li}_{1.160} \mathrm{Al}\right)$.

It should be noted that the width of the actual Li solubility range of the $\beta$ phase is expected to be larger than the 4.8 at. $\%$ determined by the potentiostatic charge counting done during lithiation only: the range is expected to extend to lower concentrations during delithiation due to the overvoltage required to move the phase boundary (and to even lower concentrations if the nucleation of the $\alpha$ phase needs to take place). Clarification has been made by holding a fully lithiated $\mathrm{Al}$ foil at $375 \mathrm{mV} v \mathrm{vs} \mathrm{Li} / \mathrm{Li}^{+}$, where only desaturation of the $\beta$ phase is occuring (i.e., no $\beta$ to $\alpha$ phase transformation). This lower boundary of the Li content in the $\beta$ phase is calculated to be 47.8 at. \% (Figure 1b) instead of the 48.9 at. \% obtained from the charge counting experiment at $250 \mathrm{mV}$, yielding a wider solubility range of 5.9 at. \% at room temperature. We note that this number has been examined by a control experiment that replicates the potentiostatic charge counting steps using a coin cell. The results are provided in Figure S2, in which consistency can be clearly observed, supporting the reliability of the obtained data used for characterizing the solubility range. Still the range is lower than the 8.2 at. \% determined by coulometric titration method at elevated temperature. ${ }^{[19]}$ The solid 
solution region is bounded by $\mathrm{Li}_{1.160} \mathrm{Al}$ and $\mathrm{Li} 0.916 \mathrm{Al}$, corresponding to specific capacities of $1152 \mathrm{mAh} \mathrm{g}^{-1}$ and $910 \mathrm{mAh} \mathrm{g}^{-1}$, respectively. Therefore, this solubility range should contributes to a specific capacity of $\sim 242 \mathrm{mAh} \mathrm{g}^{-1}(\sim 21 \%$ of the full capacity), normalized to Al, in agreement with the integrated reversible capacity of the non- $\alpha / \beta$ phase transformation seen in $\mathrm{CV}$.
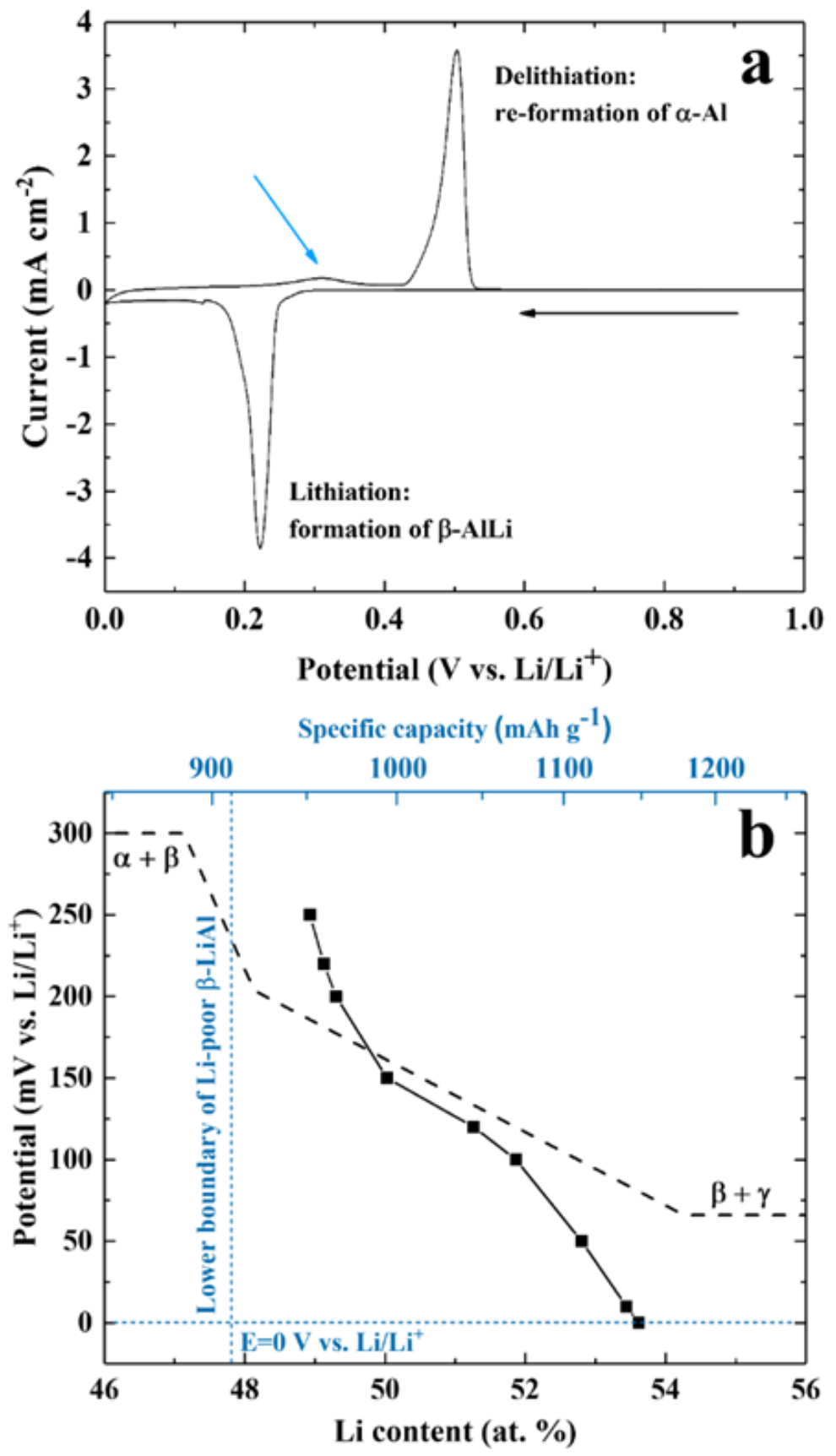
Figure 1. (a) Typical cyclic voltammogram of a $20 \mu \mathrm{m}$ thick Al foil obtained at a scan rate of $0.01 \mathrm{mV} \mathrm{s}^{-1}$ (2nd cycle). The black and blue arrows indicate the scan direction and the unclarified delithiation bump, respectively. (b) Potentiostatic charge counting data obtained from $0.25 \mathrm{~mm}$ thick $\mathrm{Al}$ foils covering a potential range from $250 \mathrm{mV}$ to $0 \mathrm{mV}$ vs. $\mathrm{Li} / \mathrm{Li}^{+}$at room temperature, with the possible lower limit of the solubility range specified. 
The solubility range of a partly prelithiated Al foil is then characterized by a series of designated cyclic voltammetry experiments. To begin with, the potential range is fixed to be between $0.4 \mathrm{~V}$ and $0.1 \mathrm{~V}$ vs. $\mathrm{Li} / \mathrm{Li}^{+}$, such that the formed $\beta$-LiAl is not delithiated beyond the solubility range and maintains its crystal structure. As illustrated in Figure $2 \mathrm{a}$, when the potential is decreased from the equilibrium potential $\left(\sim 0.35 \mathrm{~V} \mathrm{vs.} \mathrm{Li} / \mathrm{Li}^{+}\right)$, a quasi-linear increase of the reduction current can be observed, agreeing with the Geronov's rule that the speed of the phase boundary propagation rate should be linearly correlated with the driving force (i.e., overpotential). ${ }^{25}$ Here, the non-linear regime between $\sim 0.32 \mathrm{~V}$ and $0.4 \mathrm{~V}$ vs. $\mathrm{Li} / \mathrm{Li}^{+}$should refer to the $\mathrm{Li}$ solubility range of the $\beta$ $\mathrm{LiAl}$, which is the only possible origin of reversible capacity. The lithiation kinetics generally follow the one-dimensional thickening process (i.e., the geometry of the DealGrove model, ${ }^{8}$ but with propagation-limited kinetics) in the case of bulk Al foils. During the $\beta$ phase growth, a Li concentration gradient in the $\beta$ phase is required to keep the $\mathrm{Li}$ flux from the electrolyte to the phase interface and to enable continuous phase propagation. Therefore, the electrochemical driving force and the $\mathrm{Li}$ diffusion are of vital importance because of their strong effect on the phase propagation and the $\mathrm{Li}$ concentration gradient, which determines the the Li atom flux through the $\beta$-LiAl to the $\alpha / \beta$ phase interface. Accordingly, a narrower potential window and a slow scan rate should be chosen to ensure a null propagation of the phase boundary and a sufficient time for Li to diffuse within the $\beta$-LiAl. The CV scan rate was initially set at $0.1 \mathrm{mV} \mathrm{s}^{-}$ ${ }^{1}$, but the obtained cyclic voltammograms in Figure S3 indicate that the (de-)saturation of the $\beta$-LiAl seems to be limited by the Li diffusion. 
Consequently, Figure $2 \mathrm{~b}$ compares the cyclic voltammograms obtained at a 10 times slower scan rate (i.e., $0.01 \mathrm{mV} \mathrm{s}^{-1}$ ) of the $\mathrm{Al}$ foil with various prelithiation depths. It can be clearly seen that the deeper the prelithiation, the larger the CV area (i.e., capacity, energy stored, etc.) is, suggesting that the prelithiated $\beta$ phase is cycled within its solubility range. Otherwise, all cases would yield a similar CV shape if the currents were contributed by the propagation of the phase interface (i.e., growth of more $\beta$-LiAl). As shown in Figure 2c, although the areal capacities exhibit a perfect linearity vs. nominal lithiation depth, a value of $\sim 2 \mu \mathrm{m}$ instead of the coordinate origin is achieved by extrapolating the linear fit towards to the left. This observation is indicative of the SEI formation, and thus the actual lithiation depth is smaller by a constant value, compared to the nominal one calculated from the electrical charge. Quantitatively, the capacity estimated from the Al foil with a nominal lithiation depth of $20 \mu \mathrm{m}\left(0.041 \mathrm{mAh} \mathrm{cm}{ }^{-2}\right)$ is slightly lower than a half and one fourth of those from the ones with the $40 \mu \mathrm{m}(0.089$ $\left.\mathrm{mAh} \mathrm{cm}^{-2}\right)$ and the $80 \mu \mathrm{m}\left(0.181 \mathrm{mAh} \mathrm{cm}^{-2}\right)$, respectively. If taking into consideration the electrical charge that is consumed by SEI formation, perfect two-fold relationships among them can be expected. 

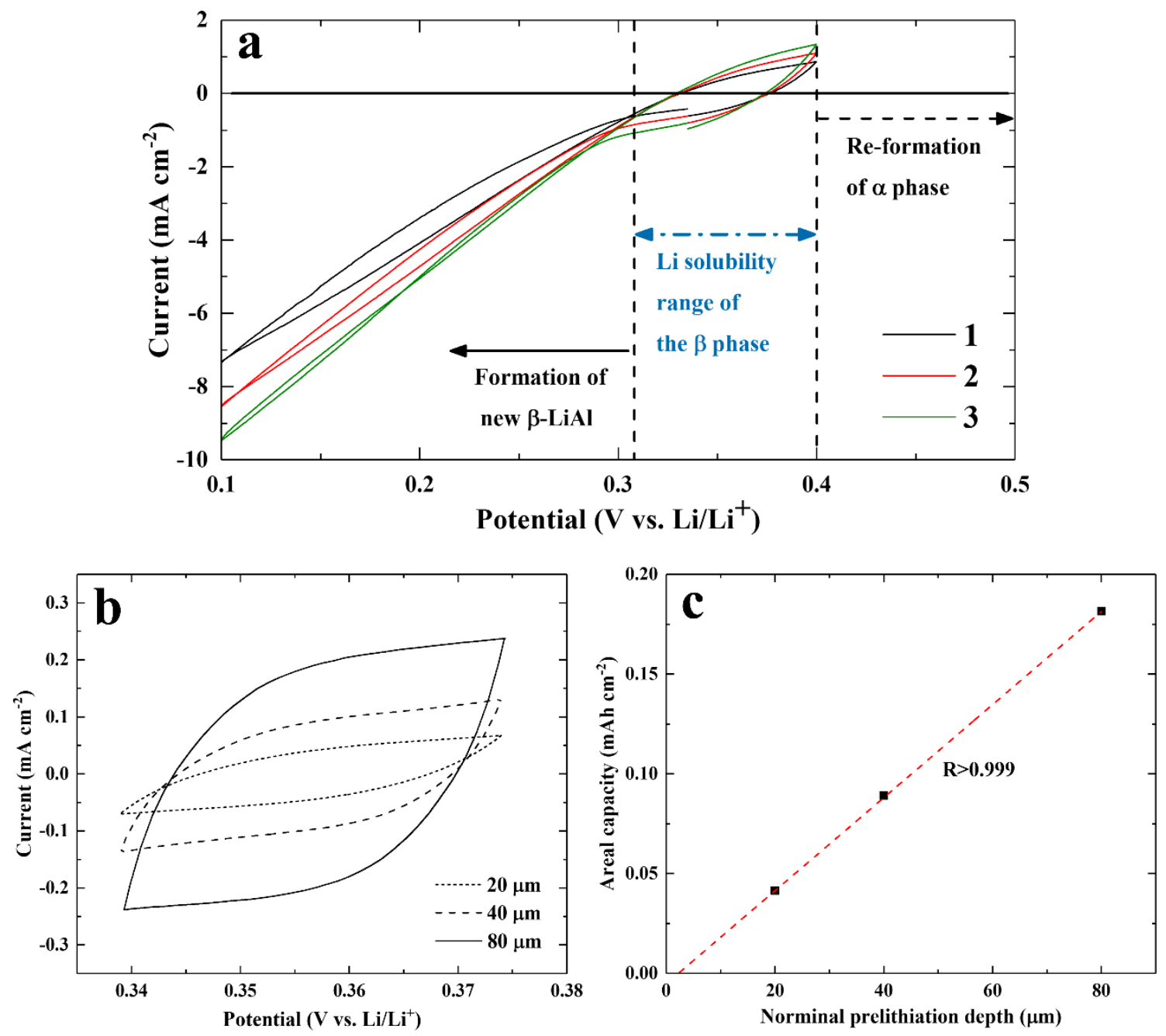

Figure 2. (a) The CV scans between $0.4 \mathrm{~V}$ and $0.1 \mathrm{~V}$ vs. $\mathrm{Li} / \mathrm{Li}^{+}$for the prelithiated $100 \mu \mathrm{m} \mathrm{Al}$ foils at a scan rate of $0.1 \mathrm{mV} \mathrm{s}^{-1}$; the nominal prelithiation depth is $20 \mu \mathrm{m}$ based on the calculation of electric charge. (b) The CV cycling at $0.01 \mathrm{mV} \mathrm{s}^{-1}$ within the determined $\mathrm{Li}$ solubility range while maintaining the $\beta$ - $\mathrm{LiAl}$ structure for the $\mathrm{Al}$ foils with various nominal prelithiation depth. (c) The areal capacity integrated from the cyclic voltammograms as a function of the nominal prelithiation depth. 


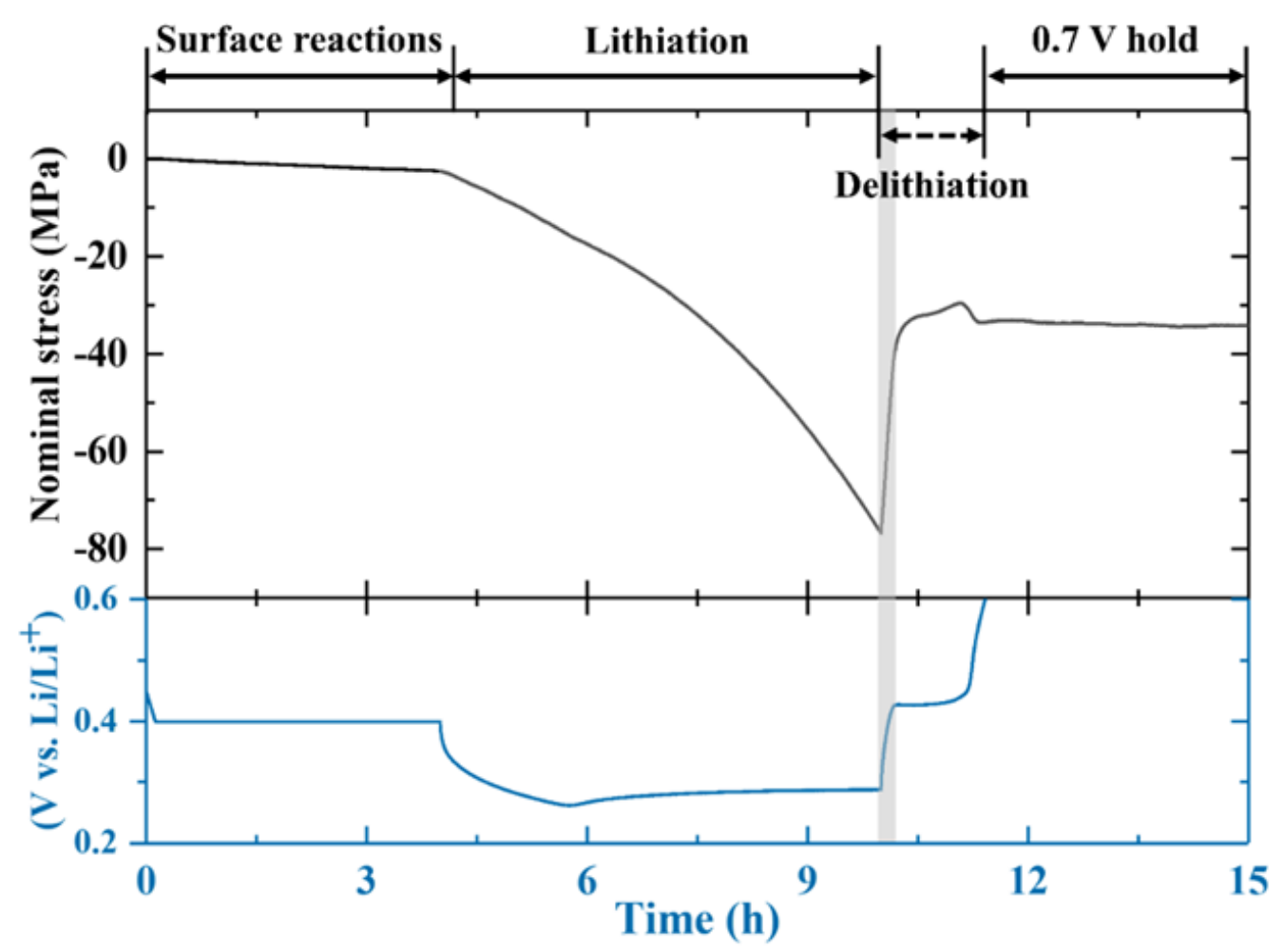

Figure 3. Nominal stress as a function of time coupled with potential responses for the initial cycle with a $\sim 40 \%$ lithiation depth. The shaded area refers to the solubility range of the $\beta$ phase. The same plot for the second cycle is provided in the Supplementary Information as Figure S4. 


\section{Solubility Range Characterized by in situ Stress Measurement}

In addition to the electrochemical characterizations, in situ substrate curvature tests were also conducted using a cell that is designed to quantify mechanical changes in the active material during cycling. ${ }^{26}$ Figure 3 shows a partial electrochemical cycle of such a setup. The cell is assembled using an as-sputtered Al thin film on a flat substrate as the working electrode and was held at $0.4 \mathrm{~V}$ vs. $\mathrm{Li} / \mathrm{Li}^{+}$at a constant temperature, such that surface reactions are minimized at the higher potential regime. With the initial state defining the zero-stress, SEI formation and oxides contribute to a less than $2 \mathrm{MPa}(<5 \%$ of the maximum) nominal stress after 4 hours when the potential is held at $400 \mathrm{mV}$ vs. $\mathrm{Li} / \mathrm{Li}^{+}$. Yet, it still takes $\sim 2$ hours for the Al film electrode to be nucleated, as indicated by the potential dip at $\sim 5.8$ hours (also described elsewhere). ${ }^{27}$ This can be explained by the finite thickness of the $\mathrm{Al}$ thin film $(\sim 420 \mathrm{~nm})$, of which the native oxide occupy several tens of nanometers. The oxide lithiation together with the initial SEI formation at the lower potential regime should be responsible for this long nucleation time, and give a nominal stress level of $\sim 20 \mathrm{MPa}$. Once the nucleation of $\beta$-LiAl occurs, the nearly linear buildup of compressive stress with a higher slope is observed during lithiation. As seen from the corresponding GCD profile, the electrode potential never goes below $0.2 \mathrm{~V}$ vs. $\mathrm{Li} / \mathrm{Li}^{+}$, supporting the conclusion that the $\beta-\mathrm{LiAl}$ is the only lithiated phase here as $\mathrm{Li}$ rich phases often require very low potentials and possibly high temperatures. ${ }^{17}$

When the $\mathrm{Al}$ film reaches the end of a partial lithiation of $\sim 40 \%$ (i.e., $\sim 4$ hours after nucleation), the lithiated $\beta$-LiAl should be saturated to some extent as overpotentials are often required to facilitate the propagation of the phase boundary. Moving to the 
delithiation, a rapid increase of the stress towards a tensile state is observed as soon as the current direction is reversed. The Li concentration in the $\beta$ phase must approach its minimum to prompt the phase transition from $\beta$-LiAl to $\alpha-A l$. This sudden stress jump at the beginning of lithiation is likely a result of desaturating the solubility range within the formed $\beta$-LiAl, as the overall volume change may strain the substrate more significantly than the two-phase coexistence where the stress is restricted to a volume near the $\alpha / \beta$ interface. The decrease in Li content from Li-rich $\beta$-LiAl to Li-poor $\beta$-LiAl shifts the lattice parameter by 0.03 angstrom, corresponding to a volume contraction of $\sim 1.4 \%$ and causing this substantial stress change. ${ }^{10}$ This solubility feature was also acknowledged by a previous study, but for a fully lithiated Al film instead of a partly lithiated one. ${ }^{9}$ Interestingly, the stress data of the previous study is consistent with the $\beta$-LiAl of a partly lithiated solid Al film being (de-)saturated without propagating the phase boundary in this work. Subsequently, a nanoporous $\alpha$-Al matrix will be created by further removing the $\mathrm{Li}$ atoms, like other dealloying processes,${ }^{28}$ thereby giving a stable stress signal from 11 hours. The second cycle of the stress cell in Figure S4 shows generally similar features, but with a more pronounced linear buildup of the compressive stress during lithiation due to less pronounced surface reactions. 


\section{Fabrication and Characterization of the Bilayer Al-based Electrode}

If the cycling capacity is limited to the $\mathrm{Li}$ solubility range of the prelithiated $\beta$-LiAl layer on top of an $\mathrm{Al}$ foil, it may be conjectured that the $\alpha / \beta$ interface will not move during charge-discharge, leading to long-term cycling capabilities. To prove this theory, SEM images were taken to help observe a partially (de-)lithiated Al foil. The prelithiation is done by initiating the potential at $10 \mathrm{mV}$ for 15 minutes to achieve a homogenous nuclei distribution covering the whole electrode surface, ${ }^{29}$ and then holding at $150 \mathrm{mV}$ to allow a one-dimensional phase boundary propagation until the amount of charge that is sufficient to lithiate a certain depth. ${ }^{25}$

Figure 4 provides the cross-sectional view of a partially lithiated Al foil, where a darker color refers to the $\beta$ - $\mathrm{LiAl}$ due to its lower electron density, resulting in a smaller number of the backscattered electrons. The $\beta$-LiAl layer at the cross section exhibits a continuous, but imperfectly flat interface due to the random nucleation that is evidenced in a previous study. ${ }^{8}$ From a macroscopic view, the surface of the Al foil electrode is covered with a layer of the dark $\beta$-LiAl while the back side exhibits the typical metallic features of aluminum.

These observations demonstrate the opportunity for solid-state bilayer Al-based anodes, i.e., growing a continuous layer of the $\beta$ - $\mathrm{LiAl}$ (active material) on the surface of an $\mathrm{Al}$ foil (current collector). The idea here is to realize the Li solubility range without propagating the phase boundary and growing more $\beta$-LiAl at the consumption of $\alpha$-Al. From a thermodynamic point of view, one may argue that the inserted Li atoms might preferentially drive the phase interface to lithiate more fresh $\mathrm{Al}$ underneath when the two phases coexist rather than saturating the $\beta$ 
phase on top of the electrode. However, the formation of additional $\beta$-LiAl will be accompanied by intrinsic barriers (e.g. mechanical strain, grain orientation, nucleation), ${ }^{30}$ causing overpotentials that largely prevent instantaneous phase transformation.

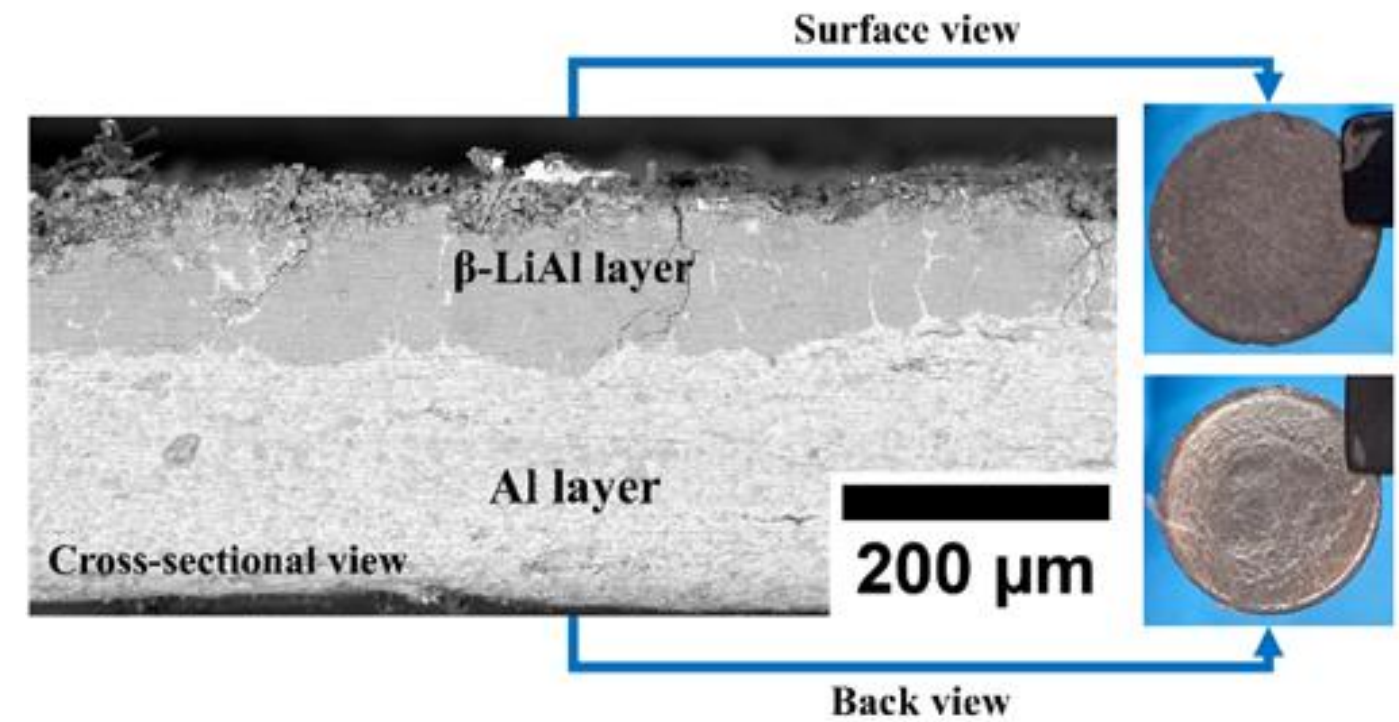

Figure 4. Cross-sectional SEM image taken for a partly lithiated Al foil using a $90^{\circ}$ sample holder at the magnification of $200 \times$. The macroscopic views of the electrode surface and the back side are also shown. 


\section{Full Cell Demonstration of the Bilayer Al-based Anode}

A full cell is used to assess its performance using such an Al-based anode and a commercially available $\mathrm{LiFePO}_{4}$ cathode. The cell structure and the conditions are schematically illustrated in Figure 5a. The capacity contributed by the solubility range of the prelithiated $\beta$-LiAl is optimized to surpass the overall capacity of the cathode. The same $\mathrm{Al}$ foil $(0.25 \mathrm{~mm})$ is prelithiated to form the bilayer structure: The upper layer of $0.1 \mathrm{~mm}$ is trainsformed to the $\beta-\mathrm{LiAl}$ and the remaining $0.15 \mathrm{~mm}$ functions as the current collector. The ideal capacity contributed by the solubility range is calcuated to be $\sim 6.5 \mathrm{mAh} \mathrm{cm}^{-2}$ using the value of $1152 \mathrm{mAh} \mathrm{g}^{-1}$ proposed in this study. However, the value can hardly be achieved because the overall $\alpha / \beta$ coexistance still remains for the bilayer electrode, and any localized overlithiation or overpotential can result in the phase boundary movement. Therefore, we start with a high N/P ratio of 6.5 , i.e., the

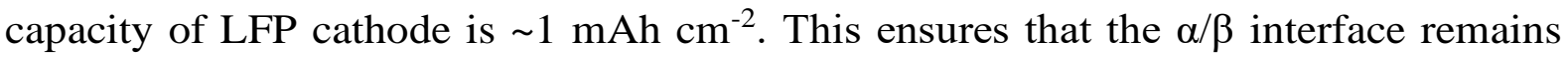
largely unperturbed. 


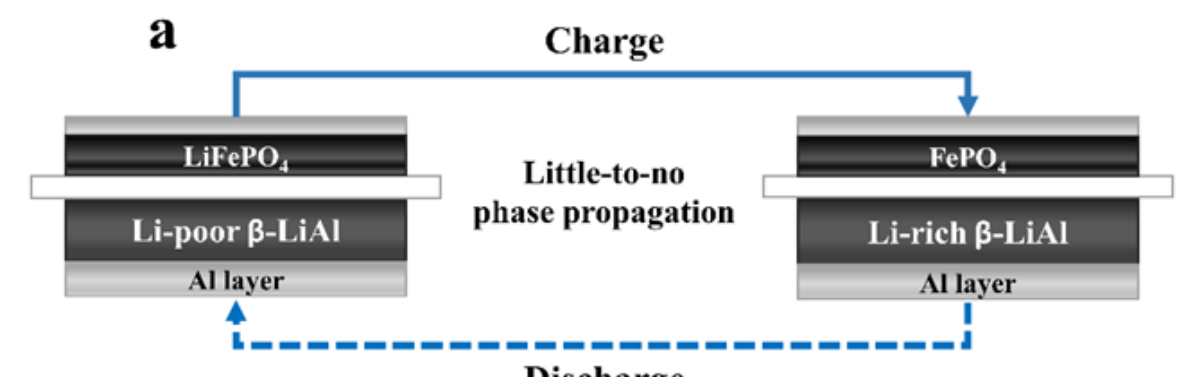

Discharge

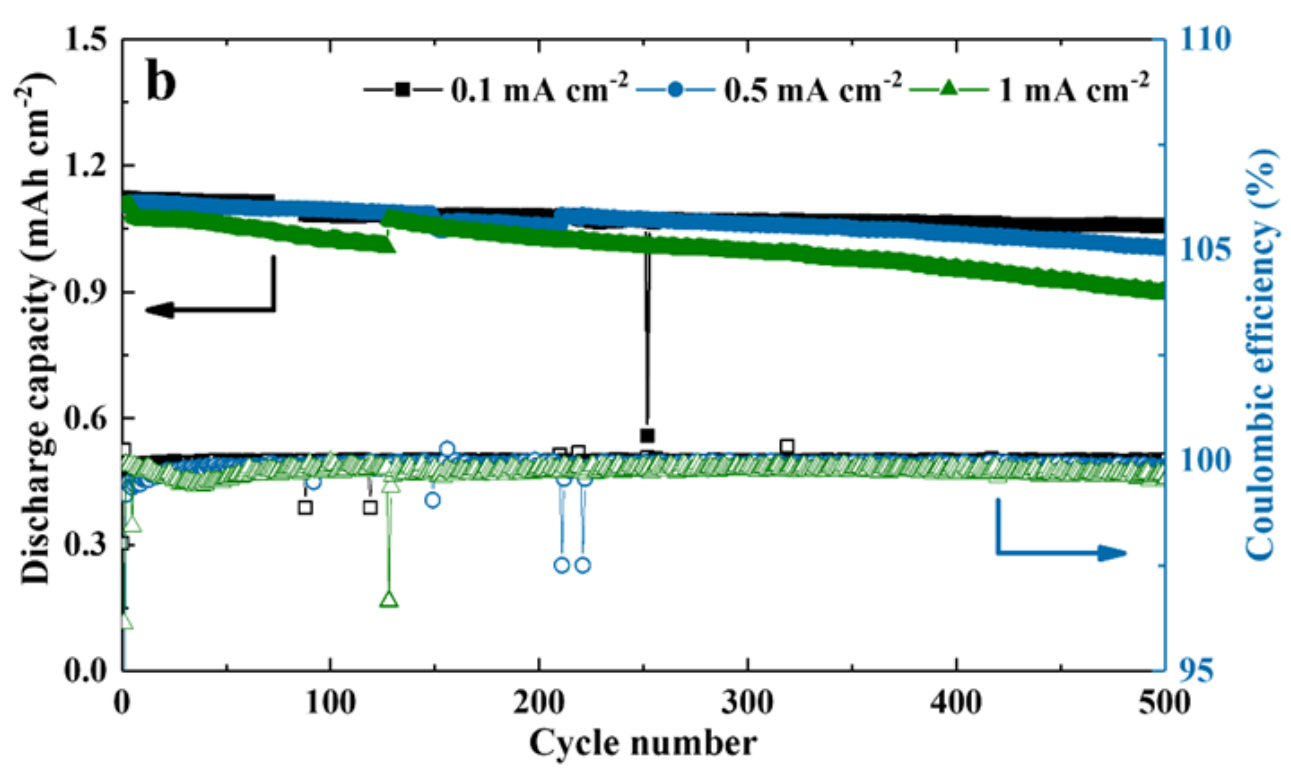

Figure 5. (a) Schematic illustrations a novel full cell design using the $\beta$-LiAl grown on an $\mathrm{Al}$ foil as anode and a commercial LFP cathode. (b) The cycling performance obtained at a current density of $0.1 \mathrm{~mA} \mathrm{~cm}^{-2}$ when the cathode capacity is comparable to the one contributed by the solubility range of the $\beta$-LiAl anode.

As shown in Figure 5b, the assembled full cell exhibits the excellent cycle lives with $95.8 \%, 90.9 \%$, and $81.5 \%$ discharge capacity retentions after 500 cycles for the current densities of $0.1,0.5$, and $1 \mathrm{~mA} \mathrm{~cm}^{-2}$, respectively. At the rates slower than $0.5 \mathrm{C}$, the capacity retentions are equivalent to $>99.99 \%\left(0.1 \mathrm{~mA} \mathrm{~cm}^{-2}\right)$ and $>99.98 \%\left(0.5 \mathrm{~mA} \mathrm{~cm}^{-}\right.$ ${ }^{2}$ ) per cycle. Although the cycling performance seems to be negatively affected by a faster rate, it already outperforms most of the commercially available batteries with $99.96 \%$ cyclic capacity retention at $1 \mathrm{~mA} \mathrm{~cm}^{-2}$ (1C). It should also be noted that the 
cell has been functioning well for more than 18 months at the time when this article is submitted.

Figure 6a depicts the GCD curves at increasing cycle numbers of Figure 5b, where similar features can be observed except the initial cycle. From these, the solubility range is only partly engaged in the first cycle, resulting in limited phase boundary movement. As cycling continues, the cell enters a balanced state where the by the solubility range of the $\beta$-LiAl layer mostly contribues to the capacity of the anode with little-to-no phase propagation. This is consistent with the assumption that there should not be any significant changes in the $\mathrm{Al}$ electrode, and the $\beta$-LiAl is still intact during cycling. Figure $6 \mathrm{~b}$ provides direct evidence in this regard: As observed, the bilayer structure of the $\mathrm{Al}$ electrode still remains after around 250 cycles (backside is metallic Al), though the lateral expansion at the circumference and some creases can be observed on the backside due to the large stress change during (de-)saturation of the $\beta$-LiAl. ${ }^{9}$ When the cell is reassembled using the same electrodes, the cycling performance does not seem to be affected by mechanical deformation, delivering the same capacity as before. 

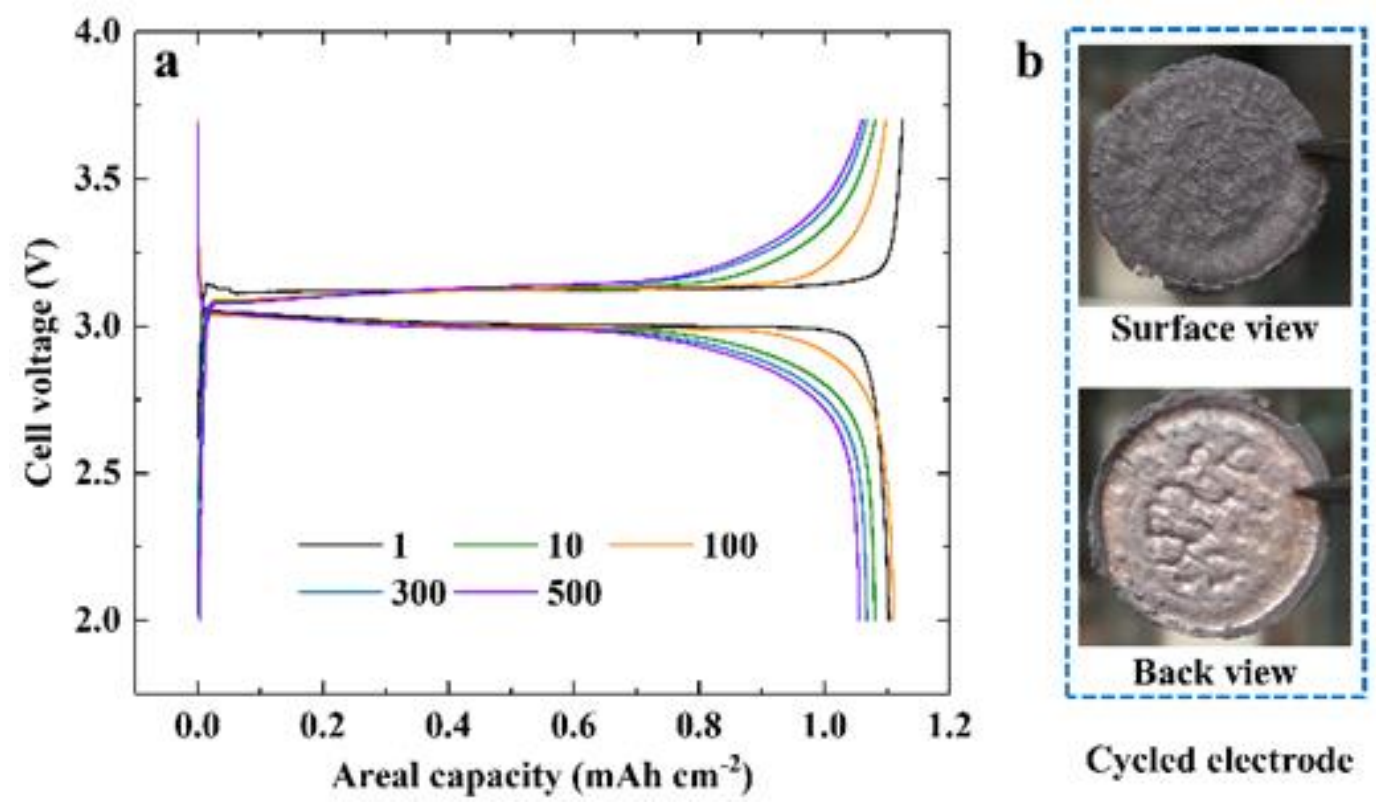

Figure 6. (a) Galvanostatic charge-discharge curves at different cycles. (b) The macroscopic views of the electrode surface and the back side after cycling, in addition to the ones presented before.

Although the presented results already exhibit readiness in commcericlaization, it is relevant to question the high $\mathrm{N} / \mathrm{P}$ ratio or the low volumetric capacity of the $\beta$-LiAl on Al electrode. Optimization efforts are required from an engineering point of view to explore the best proportion between the $\beta$-LiAl layer and the metallic Al layer, as well as the N/P ratio. For instance, a quick demonstration is provided in the Supplementry Information (Figure S5) that the concept is successful for commcerially available Al foil (0.18 mm; Toyo Aluminum K.K.). Here the prelithiation depth is done in a different way: The upper layer of $0.06 \mathrm{~mm}$ is transformed to the $\beta$ - $\mathrm{LiAl}$ and the remaining 0.12 $\mathrm{mm}$ functions as the current collector. Both layers are thinner than the ones used in Figure 5, yet the cycling performance is hardly affected (500 stable cycles) when pairing this electrode with the same LFP cathode. In this case, the capacity of the solubility 
range is calculated to be $\sim 3.9 \mathrm{mAh}^{-2}$, giving a smaller N/P ratio of $\sim 3.9$ that can hopefully be further minimized. 


\section{CONCLUSIONS AND PERSPECTIVES}

Cumulatively, these results exhibit excellent prospects and readiness for commercialization since a simple rolling methods for the prelithiation of $\mathrm{Al}$ and for the processing of $\mathrm{LiAl}$ are available. Furthermore, these observations explain the origin of the anomalous success of some cells with $\mathrm{Al}$ anodes mentioned in the literature. For full cells where the capacity of the cathode is small compared to the capacity stemming from the Li solubility range in the $\beta$ phase, an indefinite cycle life can be expected for the anode. Similarly, if the mismatch between the electrode capacities is small, then only a minimal propagation of the $\beta$ phase is expected. When there is an excess of $\alpha$ phase in the foil, the cell will be able to cycle for a very long time because the pristine $\alpha$ phase cannot be consumed by lithiation since the capacity of the cathode is limited. Recent demonstrations of LCO-Al by Hongyi $\mathrm{Li}$ et al. are fully consistent with this interpretation, ${ }^{21}$ although the influence of the mechanical strength of the pristine $\alpha-\mathrm{Al}$ underlayer merits continued investigation and optimization. While the electrochemical formation of the $\alpha / \beta$ layered structure may be preferred experimentally due to the precise control of prelithiation depth, recent work by Ryu et al. suggests that metallurgical bonding may be an easy way of forming an ideal anode. ${ }^{13}$

$\mathrm{LiAl}$ is an attractive candidate for Li-based anodes in many applications, given its excellent cycling performance, low potential and modest to high capacity figures - about $242 \mathrm{mAh} \mathrm{g}^{-1} \mathrm{Al}$ for high cycling stability as suggested here, up to $1152 \mathrm{mAh} / \mathrm{g}^{-1} \mathrm{Al}$ for few-cycle batteries. Furthermore, considering the prospects for simple foil-based construction, the solid-state layered Al-based anode provides a low barrier to market 
entry if manufacturing, tooling, and labor costs are comprised. Even aside from a foil design (i.e., $\geq 0.1 \mathrm{~mm}$ ), the metallurgical opportunities afforded by Al open up pathways for high performance 3D architectures, as already indicated by novel IdEA platforms explored by others. ${ }^{31}$ From the end-of-life perspective, a piece of metallic foil might offer alternative options for recyclers and waste processors who are concerned about the challenges of today's standard composite electrode designs and the mix of materials therein. ${ }^{32}$ 



\section{Acknowledgements}

This work was supported by grants from the Research Grants Council (PolyU 252166/17E) of the Hong Kong Special Administrative Region, China and by the internal project "Electrochemical Energy Storage Systems" (1-ZVD2) of the Hong Kong Polytechnic University (PolyU). T. Zheng is thankful to Mr. Tolga Akcay and Dr. Holger Geßwein from the IAM-WBM of Karlsruhe Institute of Technology (KIT) for running the cycling test and for conducting $\mathrm{x}$-ray diffraction experiment. The authors are also grateful to Toyo Aluminum K.K., the Toyal group for providing Aluminum foil samples. 


\section{Reference}

1. Rao, B.; Francis, R.; Christopher, H., Lithium - aluminum electrode. Journal of The Electrochemical Society 1977, 124 (10), 1490-1492.

2. Baranski, A.; Fawcett, W., The Formation of Lithium - Aluminum Alloys at an Aluminum Electrode in Propylene Carbonate. Journal of The Electrochemical Society 1982, 129 (5), 901-907.

3. Jow, T.; Liang, C., Lithium - Aluminum Electrodes at Ambient Temperatures. Journal of The Electrochemical Society 1982, 129 (7), 1429-1434.

4. Obrovac, M. N.; Chevrier, V. L., Alloy Negative Electrodes for Li-Ion Batteries. Chemical Reviews 2014, 114 (23), 11444-11502.

5. Kim, J. M.; Guccini, V.; Kim, D.; Oh, J.; Park, S.; Jeon, Y.; Hwang, T.; Salazar-Alvarez, G.; Piao, Y., A novel textile-like carbon wrapping for high-performance silicon anodes in lithium-ion batteries. Journal of Materials Chemistry A 2018, 6 (26), 12475-12483.

6. Tahmasebi, M. H.; Kramer, D.; Mönig, R.; Boles, S. T., Insights into Phase Transformations and Degradation Mechanisms in Aluminum Anodes for Lithium-Ion Batteries. Journal of The Electrochemical Society 2019, 166 (3), A5001-A5007.

7. Huang, T. S.; Brittain, J. O., The mechanical behavior of $\beta$-LiAl. Materials Science and Engineering 1987, 93, 93-97.

8. Zheng, T.; Kramer, D.; Tahmasebi, M. H.; Mönig, R.; Boles, S. T., Improvement of the Cycling Performance of Aluminum Anodes through Operando Light Microscopy and Kinetic Analysis. ChemSusChem 2020, 13 (5), 974-985.

9. Zheng, T.; Kramer, D.; Tahmasebi, M. H.; Mönig, R.; Boles, S. T., Exploring the Reversibility of Phase Transformations in Aluminum Anodes through Operando Light Microscopy and Stress Analysis. ChemSusChem 2020, 13 (22), 5910-5920.

10. Kishio, K.; Brittain, J. O., Defect structure of $\beta$-LiAl. Journal of Physics and Chemistry of Solids 1979, 40 (12), 933-940.

11. Boles, S. T.; Tahmasebi, M. H., Are Foils the Future of Anodes? Joule 2020, 4 (7), 1342-1346.

12. Gogotsi, Y.; Simon, P., True Performance Metrics in Electrochemical Energy Storage. Science 2011, 334 (6058), 917.

13. Ryu, J.; Kang, J.; Kim, H.; Lee, J. H.; Lee, H.; Park, S., Electrolyte-mediated nanograin intermetallic formation enables superionic conduction and electrode stability in rechargeable batteries. Energy Storage Materials 2020, 33, 164-172.

14. Kim, Y.; Ha, K.-H.; Oh, S. M.; Lee, K. T., High-Capacity Anode Materials for Sodium-Ion Batteries. Chemistry - A European Journal 2014, 20 (38), 11980-11992.

15. Guidotti, R. A.; Masset, P. J., Thermally activated ("thermal") battery technology: Part IV. Anode materials. Journal of Power Sources 2008, 183 (1), 388-398.

16. McAlister, A. J., The Al-Li (Aluminum-Lithium) system. Bulletin of Alloy Phase Diagrams 1982, 3 (2), 177-183.

17. Ghavidel, M. Z.; Kupsta, M. R.; Le, J.; Feygin, E.; Espitia, A.; Fleischauer, M. D. J. J. o. T. E. S., Electrochemical Formation of Four Al-Li Phases ( $\beta$-AlLi, Al2Li3, AlLi2- x, Al4Li9) at Intermediate Temperatures. 2019, 166 (16), A4034-A4040.

18. Qin, B.; Diemant, T.; Zhang, H.; Hoefling, A.; Behm, R. J.; Tübke, J.; Varzi, A.; Passerini, S., Revisiting the Electrochemical Lithiation Mechanism of Aluminum and the Role of Li-rich Phases (Li1+xAl) on Capacity Fading. ChemSusChem 2019, 12 (12), 2609-2619. 
19. Ji, B.; Zhang, F.; Sheng, M.; Tong, X.; Tang, Y., A Novel and Generalized Lithium-lon-Battery Configuration utilizing Al Foil as Both Anode and Current Collector for Enhanced Energy Density. Advanced Materials 2017, 29 (7), 1604219.

20. Zhang, M.; Xiang, L.; Galluzzi, M.; Jiang, C.; Zhang, S.; Li, J.; Tang, Y., Uniform Distribution of Alloying/Dealloying Stress for High Structural Stability of an Al Anode in High-Areal-Density LithiumIon Batteries. Advanced Materials 2019, 31 (18), 1900826.

21. Li, H.; Yamaguchi, T.; Matsumoto, S.; Hoshikawa, H.; Kumagai, T.; Okamoto, N. L.; Ichitsubo, T., Circumventing huge volume strain in alloy anodes of lithium batteries. Nature Communications 2020, 11 (1), 1584.

22. Hudak, N.; Huber, D., Nanostructured lithium-aluminum alloy electrodes for lithium-ion batteries. ECS Transactions 2011, 33 (24), 1-13.

23. Wen, C. J., Thermodynamic and Mass Transport Properties of "LiAl". Journal of The Electrochemical Society 1979, 126 (12), 2258.

24. Wen, C. J.; Weppner, W.; Boukamp, B. A.; Huggins, R. A., Electrochemical investigtion of solubility and chemical diffusion of lithium in aluminum. Metallurgical Transactions B 1980, 11 (1), 131-137.

25. Geronov, Y.; Zlatilova, P.; Staikov, G., The secondary lithium-aluminium electrode at room temperature: II. Kinetics of the electrochemical formation of the lithium-aluminium alloy. Journal of Power Sources 1984, 12 (2), 155-165.

26. Choi, Z.; Kramer, D.; Mönig, R., Correlation of stress and structural evolution in Li4Ti5012-based electrodes for lithium ion batteries. Journal of Power Sources 2013, 240, 245-251.

27. Wang, C.; Meng, Y.; Ceder, G.; Li, Y., Electrochemical Properties of Nanostructured Al1- x Cu x Alloys as Anode Materials for Rechargeable Lithium-Ion Batteries. Journal of the Electrochemical Society 2008, 155 (9), A615-A622.

28. Chen, Q.; Sieradzki, K., Spontaneous evolution of bicontinuous nanostructures in dealloyed Libased systems. Nature Materials 2013, 12, 1102.

29. Geronov, Y.; Zlatilova, P.; Staikov, G., Electrochemical nucleation and growth of $\beta$-Lial alloy in aprotic electrolyte solutions. Electrochimica Acta 1984, 29 (4), 551-555.

30. Zheng, T.; Wang, X.; Jain, E.; Kramer, D.; Mönig, R.; Seita, M.; Boles, S. T., Granular phase transformation of polycrystalline aluminum during electrochemical lithiation. Scripta Materialia 2020, 188, 164-168.

31. Heligman, B. T.; Kreder, K. J.; Manthiram, A., Zn-Sn Interdigitated Eutectic Alloy Anodes with High Volumetric Capacity for Lithium-Ion Batteries. Joule 2019, 3 (4), 1051-1063.

32. Heligman, B. T.; Manthiram, A., Elemental Foil Anodes for Lithium-lon Batteries. ACS Energy Letters 2021, 6 (8), 2666-2672. 


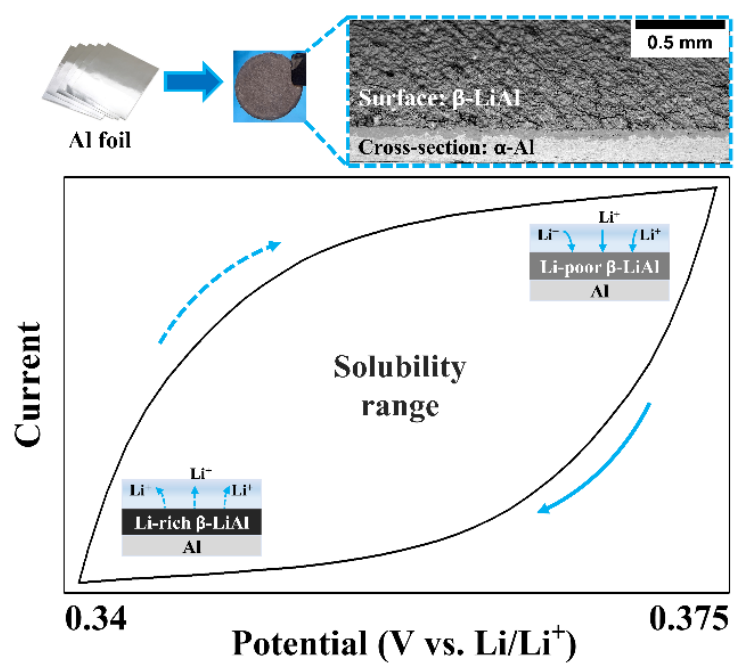

An improved understanding and manipulation of the solubility range of $\beta$-LiAl allows for fullcell devices with simple metallic foil anodes. 